\title{
Decidability of equational theories for subsignatures of relation algebra.
}

\author{
Robin Hirsch \\ Dept. of Computer Science, UCL, London \\ r.hirsch@ucl.ac.uk \\ [0000-0002-2983-4178]
}

\begin{abstract}
Let $S$ be a subset of the signature of relation algebra. Let $R(S)$ be the closure under isomorphism of the class of proper $S$-structures and let $F(S)$ be the closure under isomorphism of the class of proper $S$-structures over finite bases. Based on previous work, we prove that membership of $R(S)$ is undecidable when $S \supseteq\{\cdot,+, ;\}, S \supseteq\left\{\cdot,{ }^{\smile}, ;\right\}$ or $\smile \notin S \supseteq\{\leq,-, ;\}$, and for any of these signatures $S$ if converse is excluded from $S$ then membership of $F(S)$ is also undecidable, for finite $S$-structures.

We prove that the equational theories of $F(S)$ and $R(S)$ are undecidable when $S$ includes composition and the signature of boolean algebra. If all operators in $S$ are positive and it does not include negation, or if it can define neither domain, range nor composition, then the equational theory of either class is decidable. Open cases for decidability of the equational theory of $R(S)$ are when $S$ can define negation but not meet (or join) and either domain, range or composition. Open cases for the decidability of the equational theory of $F(S)$ are (i) when $S$ can define negation, converse and either domain, range or composition, and (ii) when $S$ contains negation but not meet (or join) and either domain, range or composition.
\end{abstract}

\section{Summary of results.}

We collect, organise are marginally extend certain results from [HH01,HJ12,Neu16]. What is offered here is a summary and a bringing together of the main results in these and other papers, a significant simplification and shortening of some of the proofs and a slight extension to the range of signatures covered by the results. We consider subsignatures $S$ of the signature of relation algebra $\left\{\leq, 0,1,-,+, \cdot, 1^{\prime}, \smile, D, D^{\prime}, R, R^{\prime}, ;, \dagger\right\}$. Signatures covered here but not elsewhere (as far as we know) include various signatures involving dual operators (rather trivially), but also signatures containing $\{\cdot,+. ;\}$, answering [Neu16, problem 3.9].

A proper $S$-structure is an $S$-structure whose elements are binary relations over some non-empty base and where constants, operators and inequalities have natural set-theoretic definitions, see (1) below. $R(S)$ denotes the class of all $S$-structures isomorphic to proper $S$-structures, $F(S)$ denotes the class of all $S$-structures isomorphic to proper $S$-structures on finite bases. 
We show, for any such signature $S$ containing $\{\leq,-, ;\}$ but not including converse, or containing $\{\cdot,+, ;\}$ or $\{\cdot,-, ;\}$ that membership of $R(S)$ is undecidable, for finite $S$-structures. If $S$ does not include converse and $S$ contains either $\{\leq,-, ;\}$ or $\{\cdot,+, ;\}$, then membership of $F(S)$ is undecidable, for finite $S$-structures. It follows that the set of quantifier-free formulas valid over each of these representation classes is undecidable. Observe that atomic formulas $a=b$ or $a \leq b$ are valid over $R(S)$ if and only if $-a=-b$, respectively $-b \leq-a$, is valid over $R(S \cup\{-\})$, and by De Morgan's law plus the duality of swapping each pair in $\left\{(\cdot,+),\left(D, D^{\prime}\right),\left(R, R^{\prime}\right),(;, \dagger)\right\}$, this holds if and only if a dual formula in a signature $S^{\prime}$ obtained from $S$ by this duality is valid over $R(S)$, hence each of these undecidablity results has a dual result for $S^{\prime}$.

If $S$ also contains the signature of boolean algebra then even the equational theory of the relevant class is undecidable (since the equational theory is contained in the quantifier-free theory, the undecidability of the equational theory entails the undecidability of the quantifier-free theory, but the converse entailment does not hold in general). On the other hand, for any signature $S$ either not including negation or including neither domain, range, composition nor their duals, the equational theories of $R(S)$ and $F(S)$ are decidable.

\section{Preliminaries.}

Let $S \subseteq\left\{\leq, 0,1,-,+, \cdot, 1^{\prime}, \smile, D, D^{\prime}, R, R^{\prime}, ;, \dagger\right\}$ be a signature. [By an extension of a convention proposed by $\mathrm{H}$. Andréka we list relations first, then booleans, then extra operators, within that we list constants then unary then binary operators, see (1) below for the intended meanings.] The boolean part of $S$ is the intersection of $S$ with $\{\leq, 0,1,-,+, \cdot\}$. An $S$-structure $\mathcal{A}$ is a finite set $A$ with a binary relation $\leq$ and elements named $0,1,1^{\prime}$, unary functions - $-{ }^{\smile}, D, D^{\prime}, R, R^{\prime}$ and binary functions $\cdot,+, ;, \dagger$ over $A$, if the symbol is included in $S$. A term of the language $L(S)$ is built from variables and constants in $S$ using operators in $S$ (note that we allow variables in terms). An atomic formula is either $s \leq t$ (if $\leq \in S$ ) or $s=t$, for terms $s, t$, the latter case is called an equation. Formulas of $L(S)$ are built from atomic formulas using first-order connectives. Given an $L(S)$-formula $\phi$, an $S$-structure $\mathcal{A}$ and a variable assignment $v:$ vars $\rightarrow \mathcal{A}$ we may evaluate $\mathcal{A}, v \models \phi$ using standard first-order semantics. For any class $\mathcal{K}$ of $S$-structures, the quantifier-free theory of $\mathcal{K}$ is the set of all quantifier-free $L(S)$ formulas $\phi$ such that for all $\mathcal{A} \in \mathcal{K}$ and all variable assignments $v:$ vars $\rightarrow \mathcal{A} \in \mathcal{K}$ we have $\mathcal{A}, v \models \phi$, similarly the equational theory of $\mathcal{K}$ is the set of equations valid over $\mathcal{K} . \phi$ is satisfiable in an $S$-structure $\mathcal{A}$ if $\mathcal{A}, v=\phi$, for some variable assignment $v$, and $\phi$ is satisfiable in some class $\mathcal{K}$ of $S$-structures if there is $\mathcal{A} \in \mathcal{K}$ and $v$ such that $\mathcal{A}, v \models \phi$.

A proper $S$-structure $\mathcal{A}$ over the base $X$ is an $S$-structure where each element of $A$ is a binary relation over $X$, where $\leq$ is set inclusion $\subseteq, \quad 0$ is the empty relation, $\cdot,+$ denote $\cap, \cup$, respectively, $1=X \times X$ (these proper structures are often called square), negation is complement in $X \times X$ (called universal 
complementation in [Neu16] ) and

$$
\begin{aligned}
1^{\prime} & =\{(x, x): x \in X\} \\
a^{\smile} & =\{(y, x):(x, y) \in a\} \\
D(a) & =\{(x, x): \exists y(x, y) \in a\} \\
D^{\prime}(a) & =-D(-a) \\
R(a) & =\{(y, y): \exists x(x, y) \in a\} \\
R^{\prime}(a) & =-R(-a) \\
a ; b & =\{(x, y): \exists z \in X(x, z) \in a \wedge(z, y) \in b\} \\
a \dagger b & =-((-a) ;(-b))
\end{aligned}
$$

(in each case, only if the inequality, constant or operator is included in $S$ ). For any proper $S$-structure $\mathcal{A}$ and subset $Y$ of the base of $A$, we write $\left.\mathcal{A}\right|_{Y}$ for the proper $S$-structure of binary relations generated by $\{a \cap(Y \times Y): a \in A\}$ in the full $S$-algebra of binary relations over $Y$. There is a boolean duality that swaps each of the pairs $\left\{(\cdot,+),\left(D, D^{\prime}\right),\left(R, R^{\prime}\right),(;, \dagger)\right\}$.

Other connectives are definable, e.g. $a \backslash b=-\left(a^{\smile} ;-b\right), A(a)=1^{\prime} \cdot D^{\prime}(-a)=$ $1^{\prime} \cdot-D(a)$, but not considered separately here. All operators considered here (other than negation) are positive. Our signatures include redundant symbols, e.g. $a \leq b \Longleftrightarrow a+b=b$ is valid over proper $\{\leq,+\}$-structures, $D(a)=1^{\prime} \cdot a ; 1$ is valid over proper $\left\{1, \cdot, 1^{\prime}, D, ;\right\}$-structures, $a \nmid b=-(-a ;-b)$ is valid over proper $\{-, \dagger, ;\}$-structures, etc. The ordering $\leq$ is only needed as a primitive relation symbol for signatures with neither - nor + . Henceforth, we work modulo interdefinability so, e.g., the signatures $\{-, ;\},\{-, \dagger\}$ are considered equal, and we may write $\{-,+\}$ for the signature of boolean algebra as $\leq, 0,1, \cdot$ are definable.

\section{Decidablity.}

Lemma 1. Let $S$ be a signature without negation. Let $s=t$ be an equation, let $k$ be the number of occurences of operators from $\{D, R, ;\}$ in $s$ or $t$. If $s=t$ is falsifiable in some proper $S$-structure $\mathcal{A}$ then it is falsifiable in some proper $S$ structure with at most $2+k$ points.

Proof. See [KNSS18, theorem 1]. Suppose $s=t$ is falsified in some proper $S$ structure $\mathcal{A}$. So, so $s, t$ denote distinct binary relations over the base $X$ of $\mathcal{A}$ and there are $x, y \in X$ such that $(x, y)$ is in the symmetric difference of $s$ and $t$, without loss $(x, y) \in s \backslash t$. Since all operators are positive, we have $(x, y) \notin t^{\mathcal{A} \mid Y}$ for any subset $Y$ of $X$. By structured induction on the term $s$, since $(x, y) \in s$, there is a finite set $F \subseteq X$ with at most $k$ points, and for any $Y$ with $\{x, y\} \cup F \subseteq$ $Y \subseteq X$ we have $(x, y) \in s^{A \uparrow_{Y}}$. Then $|F| \leq k$ and $s=t$ is falsifiable in $\mathcal{A} \uparrow_{\{x, y\} \cup F}$.

See [AB95, corollary 4, theorem 5] for the decidability of the equational theory of $R(S)$ for $S=\left\{\cdot,+, 0,1,1^{\prime}, \smile, ;\right\}$ and $S=\left\{+, 1^{\prime}, \smile, ;\right\}$.

The proof of the next lemma is trivial and omitted. 
Lemma 2. Let $S$ be a signature with neither domain, range, composition nor their duals. If a quantifier-free formula $\phi$ is satisfiable in some $S$-structure $\mathcal{A}$ over a base $X$ then there are $x, y \in X$ such that $\phi$ is satisfiable in $\mathcal{A} \uparrow_{\{x, y\}}$.

\section{Undecidability by tiling.}

For the undecidabiliy of the equational theory of $R(S)$ where $S$ contains the lattice operators and composition we use a tiling algebra. An instance $\tau$ of the version of the tiling problem considered here is a finite set of tiles and adjacencies. We may write $\operatorname{Rt}(S)=L t(T), \operatorname{Top}(S)=\operatorname{Bot}(T)$ if the adjacencies allow $S$ to be placed immediately on the left (respectively, below) $T$. It is a yes-instance if for each $T \in \tau$ there is a tiling function $f: \mathbb{Z} \times \mathbb{Z} \rightarrow \tau$ such that $f(0,0)=T$ and for all $i, j \in \mathbb{Z}, \operatorname{Lt}(f(i+1, j))=\operatorname{Rt}(f(i, j))$ and $\operatorname{Botf}(i, j+1)=\operatorname{Top}(f(i, j))$, it is a no-instance otherwise. Though hardly used here we assume there is one tile $T^{*} \in \tau$ which may be adjacent to itself vertically or horizontally but may not be adjacent to any other tile (this tile is needed for the proof of lemma 3 below, see [HH01, section 6.4], the addition of such a tile will clearly not change a yes to a no instance or vice versa).

A partial tiling is a partial function $f: \mathbb{Z} \times \mathbb{Z} \rightarrow \tau$ such that whenever $(i, j),(i+1, j) \in \operatorname{dom}(f)$ we have $\operatorname{Rt}(f(i, j))=L t(f(i+1, j))$ and similarly for vertical adjacencies within the domain of $f$. An instance $\left(\tau, T^{00}\right)$ of the deterministic tiling function consists of a finite set of tiles and adjacencies and a specified tile from the set, where there is a bijection $I: \mathbb{N} \rightarrow \mathbb{Z} \times \mathbb{Z}$ and a function $f: \mathbb{Z} \times \mathbb{Z} \rightarrow \tau$ (not necessarily a tiling function) such that $I(0)=(0,0), f(0,0)=$ $T^{00}$ and the set of tiles $T$ such that the partial map $\{(I(i), f(I(i))): i \leq n\} \cup$ $\{(I(n+1), T)\}$ is a partial tiling is either the singleton $\{f(I(n+1))\}$ or empty. It is a yes-instance of the deterministic tiling problem if and only if there is a tiling $f$ of the plane where $f(0,0)=T^{00}$. Both the tiling problem and the deterministic tiling problem are known to be undecidable [Ber66,HJ12].

From an instance $\tau$ of the tiling problem, a relation algebra $R A(\tau)$ is constructed in [HH01, section 4] (also, see the appendix, here). The following was first presented at the 1997 Relmics conference in Tunisia, proved in [HH01, theorems 3 and 4$]$.

Lemma 3. $\tau$ is a yes-instance of the tiling problem if and only if $R A(\tau)$ is a representable relation algebra.

A modified construction in [HJ12, theorem 7.6] produced a relation algebra $R A\left(\tau, T^{00}\right)$ from an instance $\left(\tau, T^{00}\right)$ of the deterministic tiling problem, and the following was proved.

Lemma 4. Let $\left(\tau, T^{00}\right)$ be an instance of the deterministic tiling problem. It is a yes-instance if and only if $R A\left(\tau, T^{00}\right)$ is a representable relation algebra.

Based on results in [HJ12,Neu16] these results may be strengthened further.

\section{Lemma 5.}


1. Let $\tau$ be an instance of the tiling problem. If the $\{\cdot,+, ;\}$-reduct of $R A(\tau)$ is in $R(\{\cdot,+, ;\})$ then $\tau$ is a yes-instance of the tiling problem.

2. Let $\left(\tau, T^{00}\right)$ be an instance of the deterministic tiling problem. If the $\{\cdot,-, ;\}$ reduct of $R A\left(\tau, T^{00}\right)$ is in $R(\{\cdot,-, ;\})$ then $\left(\tau, T^{00}\right)$ is a yes-instance of the deterministic tiling problem.

Converse implications to both parts follow from lemmas 3,4 .

Proof. Part (2) is proved in [HJ12, theorem 8.6]. For part 1, the case $S \supseteq$ $\left\{\cdot,+, 1^{\prime}, ;\right\}$ is covered in [HJ12, theorem 6.1], the case $S \supseteq\{\cdot,+, ;\}$ answers [Neu16, problem 3.9] and is proved in lemma 12, in the appendix.

Hence

Lemma 6. Membership of $R(S)$ is undecidable, for finite $S$-structures, for signatures $S \supseteq\{\cdot,+, ;\}$ or $S \supseteq\left\{\cdot,,^{-}, ;\right\}$.

\section{Undecidability by partial groups.}

The known representations of tiling algebras $R A(\tau)$ are all infinite - whether they can be adapted to provide finite representations remains unknown. For results on finite representability we may use a different construction based on partial groups, provided converse is not included in the signature. A finite partial group $\mathcal{A}=(A, e, \sqrt{A}, *)$ consists of a finite set $A$ including some element $e$, a subset $\sqrt{A} \ni e$ of $A$ and a total binary surjective function $*: \sqrt{A} \times \sqrt{A} \rightarrow A$ such that $e * a=a * e=a$, for all $a \in \sqrt{A}$. It is a yes-instance of the partial group embedding problem if there is a group $(G, e, \circ)$ where $G \supseteq A$ and for $a, b \in \sqrt{A}$ we have $a \circ b=a * b$, it is a no-instance otherwise. The partial group finite embedding problem has the same set of instances $\mathcal{A}$, it is a yes-instance if there is a finite group $(G, e, \circ)$ where $G \supseteq A$ such that $a, b \in \sqrt{A} \Rightarrow a \circ b=a * b$, otherwise it is a no instance. Both problems are known to be undecidable, see [Eva53,JV09].

From a finite partial group $\mathcal{A}$, a finite $\left\{-,+, 1^{\prime}, ;\right\}$-structure $M(\mathcal{A})$ is defined in [HJ12, section 3], whose signature contains the whole signature of relation algebra except converse is excluded. The map that sends $\mathcal{A}$ to $M(\mathcal{A})$ is shown to be a reduction of the partial group embedding problem to $R\left(\left\{-,+, 1^{\prime}, ;\right\}\right)$ and of the parital group finite embedding problem to $F\left(\left\{-,+, 1^{\prime}, ;\right\}\right)$ [HJ12, proposition 5.1]. Without $1^{\prime}$ in the signature, if $M(A) \in R(S)$ we cannot be sure that any reflexive edges $(x, x)$ belong to 1 in a proper $S$-structure $\mathcal{B}$ isomorphic to $M(\mathcal{A})$, and such points are needed in the proof of undecidability. However, $M(\mathcal{A})$ includes an atom $e_{0}$ where $e_{0} ; e_{0}=e_{0}$, so if $\mathcal{B}$ has a finite base $X$ then there must be points $x \in X$ where $(x, x) \in e_{0}^{\mathcal{B}}$, and when negation is in the signature, since it is complementation relative to $X \times X$, every reflexive edge $(x, x)$ belongs to some element of $\mathcal{B}$. These reflexive points are needed for the proof of the following to work [Neu16, theorem 2.5]. 
Lemma 7. Let $S$ be a signature without converse. Membership of $F(S)$ is undecidable when $\{\cdot,+, ;\} \subseteq S$ and membership of either $R(S)$ and $F(S)$ are undecidable when $\{\leq,-, ;\} \subseteq S$, for finite $S$-structures.

Problem 1. Is membership of $F(S)$ decidable for finite $S$-structures, when $\{\cdot, \smile, ;\} \subseteq$ $S ?$

\section{Quantifier-free Theory and Equational Theory}

Lemma 8. If $S$ does not include negation then the set of equations valid over $R(S)$ is co-NP-complete, the same holds for $F(S)$. If $S$ does not include domain, range, composition nor their boolean duals then the validity of quantifierfree formulas is co-NP-complete.

Proof. For the first part, by lemma 1 a falsifiable equation is falsifiable in an $S$ structure of size at most two plus the number of occurrences of $\{D, R, ;\}$ in the equation. To prove that the complementary problem (satisfiability of inequations problem) belongs to NP consider a non-deterministic algorithm that, given a inequality $s \neq t$, guesses a proper $S$-structure $\mathcal{A}$ whose base size is at most two plus the number of occurrences of $\{D, R, ;\}$ in $s$ or $t$, guesses two points $x, y$ in the base, and verifies that $(x, y)$ is in the symmetric difference of $s$ and $t$. Since PSAT trivially reduces to the complement of either problem in the lemma (without using any symbol from $S$ ), they are both co-NP-hard.

The proof of the second part is similar, this time based on lemma 2.

For any signatures $S$ and finite $S$-structures $\mathcal{A}$, let the diagram of $\mathcal{A}, \Delta(\mathcal{A})$ be the quantifier-free formula

$$
\bigwedge_{a \leq b} x_{a} \leq x_{b} \wedge \bigwedge_{a \notin z b} \neg\left(x_{a} \leq x_{b}\right) \wedge \bigwedge_{a \neq b \in \mathcal{A}} \neg\left(x_{a}=x_{b}\right) \wedge \bigwedge_{o \in S} o\left(\overline{x_{a}}\right)=x_{o(\bar{a})}
$$

where the first two conjunctions are only included when $S \cap\{\cdot,+\}=\emptyset, \leq$ $\in S, \quad \bar{a}$ ranges over tuples over $\mathcal{A}$ of the same arity as $o \in S$ and $\overline{x_{a}}$ is a tuple of corresponding variables. Then $\Delta(\mathcal{A})$ is satisfiable in $\mathcal{B}$ if and only if $\mathcal{A}$ embeds into $\mathcal{B}$, and since $R(S)$ and $F(S)$ are closed under substructures, $\Delta(\mathcal{A})$ is satisfiable in $R(S)$ if and only if $\mathcal{A} \in R(S)$ and $\Delta(\mathcal{A})$ is satisfiable in $F(S)$ if and only if $\mathcal{A} \in F(S)$. Hence,

Lemma 9. Let $\mathcal{K}=R(S)$ or $F(S)$. The map $\mathcal{A} \mapsto \Delta(\mathcal{A})$ is a reduction from memberhips of $\mathcal{K}$ for finite $S$-structures to the satisfiability of quantifier-free formulas over $\mathcal{K}$.

Lemma 10. The set $Q F(R(S))$ of quantifier-free formulas valid over $R(S)$ is undecidable, for signatures $S$ containing $\{\cdot,+, ;\},\{\cdot, \smile, ;\},\{\cdot,+, \dagger\},\{+, \smile, \dagger\}$ or $\{\leq,-, ;\}$. The set of quantifier-free formulas satisfiable over $F(S)$ is undecidable, for signatures $S$ without converse, but containing $\{\cdot,+, ;\},\{\leq,-, ;\}$, or $\{\cdot,+, \dagger$. 
Proof. By lemmas 6, 7 and 9.

Lemma 11. Let $S$ contain the signature of boolean algebra and include composition, let $\mathcal{K}$ be the class of all $S$-structures such that the term $1 ; x ; 1$ is a unary discriminator (i.e. $1 ; 0 ; 1=0$ and $1 ; x ; 1=1$ for $x \neq 0$ is valid in $\mathcal{K}$ ). For each quantifier-free formula $\phi$ there is an equation $s=0$, effectively computable from $\phi$, and equivalent to $\phi$ over $\mathcal{K}$.

Proof. Make the following replacements of atomic formulas in $\phi: s \leq t \mapsto s+t=$ $t, s=t \mapsto s \cdot(-t)+(-s) \cdot t=0$, so that all atomic formulas have the form $s=0$. Since $S$ contains the signature of boolean algebra, this equation is equivalent to $\phi$ over $\mathcal{K}$. We may assume that the propositional connectives in $\phi$ are $\wedge, \neg$. Replace $s=0 \wedge t=0$ by $s+t=0$ and replace $\neg(s=0)$ by $-(1 ; s ; 1)=0$.

We now restrict to algebraic signatures (signatures without $\leq$ ) and summarise the results on the equational theories of these representation classes.

Theorem 1. The equational theory of $R(S)$ is undecidable if $\{-,+, ;\} \subseteq S$, the equational theory of $R(S)$ is decidable if $S$ does not include negation, and even the quantifier-free theory is decidable if $S$ includes neither domain, range, composition nor their duals. [The open cases for decidability of equational theory of $R(S)$ are $\{-, x\} \subseteq S \subseteq\left\{-, 1^{\prime}, \smile, D, R, ;\right\}$ where $x \in\{D, R, ;\}$.]

The equational theory of $F(S)$ is undecidable if $S$ does not include converse and $\{-,+, ;\} \subseteq S$, it is decidable if either (i) negation is not included or (ii) neither domain, range, composition nor their duals are included in $S$. [The open cases are $\{-, x\} \subseteq S \subseteq\left\{-, 1^{\prime}, D, R, ;\right\}$ and $\{-, \smile, x\} \subseteq S$ where $x \in\{D, R, ;\}$.

Proof. Let $S \supseteq\{-,+, ;\}$. Let $\tau$ be an instance of the tiling problem. The term $1 ; x ; 1$ is a discriminator for the $S$-reduct of the simple relation algebra $R A(\tau)$. By lemma 11 there is an equation $s(\tau)=0$ equivalent over $\left\{R A\left(\tau^{\prime}\right)\right.$ : $\tau^{\prime}$ is an instance of the tiling problem $\}$ to $\Delta(R A(\tau))$. By lemma 9 , the map $\tau \mapsto$ $(s(\tau)=0)$ is a reduction from the tiling problem to the equational theory of $R(S)$, so this is undecidable. For cases where $S$ excludes converse, note that $1 ; x ; 1$ is a discriminator for the $S$-structure $M(\mathcal{A})$, where $\mathcal{A}$ is a partial group, so a similar argument shows that the equational theory of $F(S)$ is undecidable. If $S$ does not include negation or is disjoint from $\{D, R, ;\}$ then the equational theories of $R(S)$ and $F(S)$ are decidable, by lemma 8 .

\section{Conclusion}

The table below summarises results on the decidability of membership of $R(S), F(S)$ for finite $S$-structures, quantifier-free theories of $R(S), F(S)$, and equational theories of these classes, $u, d$, ? denote undecidable, decidable and unknown/variable, 
respectively.

\begin{tabular}{|c|c|c|c|c|}
\hline Signature & $m(R(S))$ & $m(F(S))$ & $Q F(R(S))$ & $Q F(F(S))$ \\
\hline $\bar{S} \supseteq\{\cdot,+, ;\}$ or $S \supseteq\{\cdot,+, \dagger$ & $u$ & $u$ if $\smile \notin S$ & $u$ & $u$ if $\smile \notin S$ \\
\hline$S \supseteq\{\cdot, \smile, ;\}$ or $S \supseteq\{\cdot, \smile, \dagger$ & $u$ & $?$ & $u$ & \\
\hline$\smile \notin S, S \supseteq\{\leq,-, ;\}$ & $u$ & $u$ & $u$ & $u$ \\
\hline$\left\{D, D^{\prime}, R, R^{\prime}, ;, \dagger\right\} \cap S=\emptyset$ & $d$ & $d$ & $d$ & $d$ \\
\hline Signature & \multicolumn{2}{|l|}{$\operatorname{EqTh}(R(S))$} & \multicolumn{2}{|l|}{$\operatorname{EqTh}(F(S))$} \\
\hline$\{-,+, ;\} \subseteq S, \smile \notin S$ & \multicolumn{2}{|l|}{$u$} & \multicolumn{2}{|l|}{$u$} \\
\hline$\{-,+, \smile, ;\} \subseteq S$ & \multicolumn{2}{|l|}{$\begin{array}{l}u \\
d\end{array}$} & \multicolumn{2}{|l|}{$?$} \\
\hline $\begin{array}{l}-\notin S \\
S D D^{\prime} B B^{\prime} \cdot+t \rho S-\emptyset\end{array}$ & \multirow{2}{*}{\multicolumn{2}{|c|}{$d$}} & \multirow{2}{*}{\multicolumn{2}{|c|}{$d$}} \\
\hline$\left\{D, D^{\prime}, R, R^{\prime}, ;, \dagger\right\} \cap S=\emptyset$ & & & & \\
\hline
\end{tabular}

For the membership problems, the cases not covered by the table are cases involving domain, range or duals but not composition or relative sum, cases where converse and composition are in $S$ but not intersection, or where composition is in $S$ but not converse or intersection and $S$ omits either $\leq$ or - , also the single case $S=\{\cdot, ;\}$, plus duals of these cases. For one signature under the second case $S=\{\leq,-, D, R, ;\}$ we know that $m(R(S))$ and $m(F(S))$ are both decidable [HM13], other cases remain open.

\section{Appendix: The tiling algebra $R A(\tau)$.}

Let $\tau$ be an instance of the tiling problem, recall that $T^{*} \in \tau$ is a special tile that may be adjacent to itself but to no other tile. The following construction of a relation algebra $R A(\tau)$ is from [HH01, section 4]. The boolean part of $R A(\tau)$ is finite and has the following atoms:

$A t=\left\{e_{i}, w_{i j}, c_{0 k}, c_{k 0},+1_{k},-1_{k}, T_{12}, T_{21}: i, j<3, c \in\{g, u, v\}, 1 \leq k \leq 2, T \in \tau\right\}$.

The identity is $e_{0}+e_{1}+e_{2}$, the converse of an atom $x_{i j}$ is $x_{j i}$, the converse of $+1_{k}$ is $-1_{k}$. To define composition we list the forbidden triples of atoms $F$ and then let $\alpha ; \beta=\sum\{c \in A t: \exists a \leq \alpha, b \leq \beta,(a, b, c) \notin F\}$, for $\alpha, \beta \in R A(\tau)$.

Any triple where the indices do not match is forbidden, e.g. $\left(x_{i}, y_{i^{\prime} j^{\prime}}, z_{i^{*}, j^{*}}\right)$ is forbidden if $i \neq i^{\prime}, j^{\prime} \neq j^{*}$ or $i \neq i^{*}$. Any triple of atoms $\left(e_{i}, a, b\right)$ where $a \neq b$ is forbidden. The following are also forbidden.

$$
\begin{gathered}
\left(g_{10}, g_{02}, w_{12}\right) \\
\left(S_{12}, T_{21}, 1_{1}\right) \text { any } S, T \in \tau, \text { unless } R t(S)=L t(T) \\
\left(u_{10}, g_{02}, T_{12}\right) \text { any } T \in \tau \backslash\left\{T^{*}\right\} \\
\left(v_{10}, g_{01},+1_{1}\right),\left(v_{10}, g_{01},-1_{1}\right)
\end{gathered}
$$

There are three dual rules for forbidden triples, obtained from 4,5 and 6 by swapping the subscripts 1 and 2 throughout and replacing $L t, R t$ by Bot, Top, respectively. The Peircean transforms of a triple of atoms $(a, b, c)$ may be obtained by a sequence of up to three maps $(a, b, c) \mapsto\left(c^{\smile}, b^{\smile}, a^{\smile}\right)$ or $(a, b, c) \mapsto\left(b, c^{\smile}, a^{\smile}\right)$. $F$ is the set of Peircean transforms of the triples of atoms forbidden above. 
Lemma 12. Let $\tau$ be an instance of the tiling problem and $S \supseteq\{\cdot,+, ;\}$. If $R A(\tau) \in R(S)$ then $\tau$ is a yes-instance.

Proof. Suppose $R A(\tau)$ is isomorphic to a proper $S$-structure over a base $X$. Without loss, the isomorphism is the identity map, all elements of $R A(\tau)$ are binary relations over $X$, all operators in $S$ are defined set-theoretically, but note that if $0,1^{\prime} \notin S$ we cannot assume they are represented as the empty set and identity, and for each $a \in R A(\tau)$ the element $\bar{a} \in R A(\tau)$ might not be the true complement of $a$. But $+\in S$ so $a \leq b \Longleftrightarrow a \subseteq b$. For each tile $T^{00} \in \tau$, since

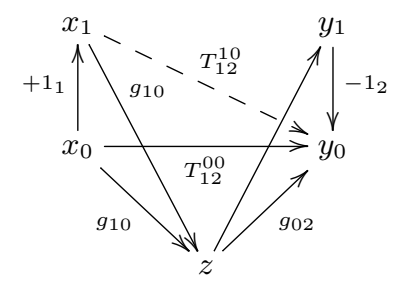

Fig. 1. If $R A(\tau) \in R\{\cdot,+, ;\}$ then points $z, \ldots, x_{-1}, x_{0}, x_{1}, \ldots, y_{0}, y_{1}, \ldots$ must exist in any representation, as shown.

$T_{12}^{00} \not \leq 0$ there are $x_{0}, y_{0} \in X$ such that $\left(x_{0}, y_{0}\right) \in T_{12}^{00}$ and $\left(x_{0}, y_{0}\right) \notin 0$, see the figure.

Since $\left(g_{10}, g_{02}, T_{12}^{00}\right)$ is not forbidden and ; is composition of binary relations, there must be $z \in X$ with $\left(x_{0}, z\right) \in g_{10},\left(z, y_{0}\right) \in g_{02}$. Since $\left(+1_{1}, g_{10}, g_{10}\right)$ is not forbidden there must be a point $x_{1}$ such that $\left(x_{0}, x_{1}\right) \in+1_{1}$ and $\left(x_{1}, z\right) \in g_{10}$. Continuing in this way there are $x_{2}, x_{3}, \ldots \in X$ where $\left(x_{i}, x_{i+1}\right) \in+1_{1},\left(x_{i+1}, z\right) \in$ $g_{10}$ for $i \in \mathbb{N}$. Since $\left(-1_{1}, g_{10}, g_{10}\right)$ is not forbidden there are also $x_{-1}, x_{-2}, \ldots \in$ $X$ where $\left(x_{i}, x_{i-1}\right) \in-1_{1}$ and $\left(x_{i-1}, z\right) \in g_{10}$, for $0 \geq i \in \mathbb{Z}$. Dually, there are points $y_{j}$ for $j \in \mathbb{Z}$ where $\left(z, y_{j}\right) \in g_{02}$ and $\left(y_{j}, y_{j+1}\right) \in+1_{2}(j \geq 0)$, $\left(y_{j}, y_{j-1}\right) \in-1_{2}(j \leq 0)$.

From $\left(x_{i}, z, y_{j}\right)$ we have $\left(x_{i}, y_{j}\right) \in g_{10} ; g_{02}=\sum_{T \in \tau} T_{12}$ and from $\alpha ; 0 ; \beta=0$ for $\alpha, \beta \in \mathbf{R A}(\tau)$ we have $\left(x_{i}, y_{j}\right) \notin 0$, for $i, j \in \mathbb{Z}$. Since $+\in S$ there is a tile $T^{i j} \in \tau$ such that $\left(x_{i}, y_{j}\right) \in T_{12}^{i j}$ and since $\in S$ this tile is unique. From $\left(x_{i}, x_{i+1}, y_{j}\right)$ we have $\left(x_{i}, y_{j}\right) \in T_{12}^{i j} \cdot\left(+1_{1} ; T_{12}^{i+1, j}\right)$ so $\operatorname{Rt}\left(T^{i j}\right)=\operatorname{Lt}\left(T^{i+1, j}\right)$ for $i \geq 0$, similarly, using $-1_{1}$ the same holds for $i<0$ and dually $\operatorname{Top}\left(T^{i j}\right)=$ $\operatorname{Bot}\left(T^{i, j+1}\right)$ for $i, j \in \mathbb{Z}$, so the map $(i, j) \mapsto T^{i j}$ is a tiling of the plane mapping $(0,0)$ to $T^{00}$. Since $T^{00} \in \tau$ was arbitrary, $\tau$ is a yes-instance of the tiling problem.

\section{References}

[AB95] H Andréka and D Bredikhin. The equational theory of union-free algebras of relations. Algebra Universalis, 33:516-532, 1995.

[Ber66] R Berger. The undecidability of the domino problem, volume 66 of Memoirs. Amer. Math. Soc., Providence, Rhode Island, 1966. 
[Eva53] T Evans. Embedability and the word problem. Journal of the London Mathematical Society, 28:76-80, 1953.

[HH01] R Hirsch and I Hodkinson. Representability is not decidable for finite relation algebras. Trans. Amer. Math. Soc., 353:1403-1425, 2001.

[HJ12] R Hirsch and M Jackson. Some undecidable problems on representability as binary relations. J. Symbolic Logic, 77(4):1211-1244, 2012.

[HM13] R Hirsch and S Mikulas. Ordered domain algebras. Journal of Applied Logic, 2013.

[JV09] M Jackson and M Volkov. Undecidable word problems for completely 0simple semigroups. Journal of Pure and Applied Algebra, 213:1961-1978, 2009.

[KNSS18] A Kurucz, I Nemeti, I Sain, and A Simon. Decidable and undecidable modal logics with a binary modality. Journal of Logic, Language and Information, 4:191-206, 2018.

[Neu16] M Neuzerling. Undecidability of representability for lattice-ordered semigroups and ordered complemented semigroups. Algebra Universalis, 76:431443,2016 . 